\title{
Compton Scattering on Light Nuclei at the High Intensity Gamma Ray Source and Electromagnetic Polarizabilities
}

\section{Mohammad W. Ahmed ${ }^{* \dagger}$}

North Carolina Central University and TUNL

E-mail: ahmedetunl.duke.edu

\begin{abstract}
A summary of the Compton scattering program at the High Intensity Gamma Ray Source (HIGS) is presented. Preliminary results of Compton scattering cross-section measurements on ${ }^{4} \mathrm{He},{ }^{2} \mathrm{H}$, and ${ }^{1} \mathrm{H}$ are presented. The determination of nucleon scalar electromagnetic polarizabilities from data form Compton scattering data on few-nucleon systems is described. Also, plans for performing Compton scattering measurements on ${ }^{3} \mathrm{He}$ with high energy resolution are discussed.
\end{abstract}

The 9th International workshop on Chiral Dynamics

17-21 September 2018

Durham, NC, USA

\footnotetext{
${ }^{*}$ Speaker.

${ }^{\dagger}$ For the Compton@HIGS Collaboration
} 


\section{Introduction}

The electromagnetic (EM) polarizabilities of the nucleon are its fundamental structure constants and have been of considerable interest for over two decades. In the classical model, the polarizabilities measure the "stiffness" response of the nucleon (a composite object) to externally applied electric or magnetic fields. In this view, the electric polarizability corresponds to the "stretching" of an extended object composed of positive and negative charges when exposed to an external electric field. The magnetic counterpart: the paramagnetic polarizability corresponds to the alignment of constituent magnetic dipoles with an externally applied magnetic field, whereas the diamagnetic contribution corresponds to a flow of charge acting to oppose the applied field. The preferred method of applying external EM fields is photon scattering from nucleons and light nuclei, such as the Compton scattering process.

In case of a spin $\frac{1}{2}$ object, such as the proton or neutron, the low-energy Compton scattering amplitude can be expanded in powers of the photon energy $(\omega)$. The proton's electric $\left(\alpha_{p}\right)$ and magnetic $\left(\beta_{p}\right)$ polarizabilities enter at order $\omega^{2}$ due to an interference with the leading Thomson amplitude. However, for a "free" uncharged neutron, there is no Thomson term, therefore, the polarizabilities enter at order $\omega^{4}$ and with smaller amplitudes. This problem is somewhat alleviated by using the deuteron or ${ }^{3} \mathrm{He}$ as effective neutron targets. ${ }^{3} \mathrm{He}$ holds a counting advantage over ${ }^{2} \mathrm{H}$, due to the $\mathrm{Z}^{2}$ dependence of the Compton cross section for light nuclei. However, these light nuclei at best provide an isospin averaged nucleon polarizabilities since the cross sections extract the sum of neutron and proton polarizabilities $\left(\alpha_{p}+\alpha_{n}, \beta_{p}+\beta_{n}\right)$. However, a precise and model independent measurement of the proton values could yield precise neutron values.

Stringent tests of the predictions of Chiral Effective Field Theories ( $\chi$ EFT) for light nuclei, which provide a link between a low-energy description of hadrons and Quantum Chromodynamics (QCD) can be pursued by precision measurements of the Compton cross sections. The $\chi \mathrm{EFT}$ calculations of the elastic Compton scattering cross sections of the deuteron and ${ }^{3} \mathrm{He}$ provide the theory framework for extracting the nucleon polarizabilities [1,2]. The current status of the polarizabilities is summarized in figure 1. This EFT extraction is based upon a global fit to proton and deuteron data sets. The most recent experimental effort which prompted this new extraction was performed at LUND [3]. It can be noted that the uncertainties for the case of the neutron are far larger than for the proton, and if the constraint of the Baldin Sum Rule (BSR) is relaxed, the uncertainties for both the proton and neutron grow considerably.

Thus far, the neutron polarizabilities extractions have predominantly employed the deuteron Compton scattering data. However, recent calculations for the case of ${ }^{3} \mathrm{He}$ have now opened another venue to pursue, i.e., Compton scattering from unpolarized ${ }^{3} \mathrm{He}$ (see the contribution from Grieshammer in these proceedings). The EFT predictions and sensitivity to the polarizability determinations for ${ }^{3} \mathrm{He}$ are discussed in detail by Griesshammer in these proceedings.

The case of the proton can be further improved by considering measurements which do not rely upon invoking the BSR, thus removing model dependence from the extraction and bringing the uncertainties for the "free" case closer to the constrained fit. These scenarios have also been calculated by [2] in the framework of Baryon $\chi \mathrm{PT}(\mathrm{B} \chi \mathrm{PT})$ [4]. These methods require the use of linearly polarized photons and the observation that at certain scattering angles (e.g., $\theta=90^{\circ}$ ) independent measurements of $\alpha_{p}$ and $\beta_{p}$ are possible due to the asymmetry in the cross sections 


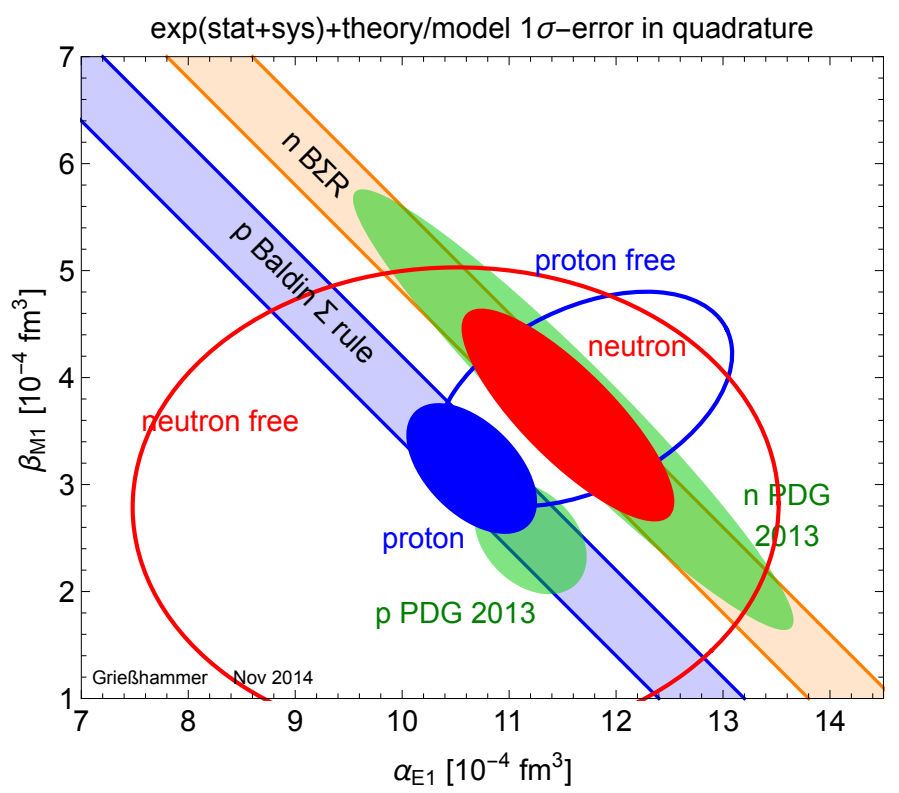

Figure 1: The current status of proton and neutron EM polarizabilities by H. Griesshammer [1, 2]. Precise and systematic EFT extractions have jogged the Particle Data Book (PDG) accepted values towards the most recent EFT extractions. The open circles ("free") are the EFT extractions without the constraint of the Baldin Sum Rule.

between counting in the plane of linear beam polarization and perpendicular to it. This technique has been proposed both at HIGS and at Mainz; these complementary programs are currently underway.

\section{Compton Scattering Setup at HIGS}

Compton scattering measurements at HIGS use an experimental setup consisting of HIGS NaI Detector Array (HINDA) [7], two large NaI detectors DIANA and BUNI, a cryogenic target [8] , and a fully digitized data acquisition system. The HIGS facility [5] uses a free electron laser to produce intense, quasi-monoenergetic, nearly $100 \%$ polarized gamma-ray beams via Compton back-scattering. Gamma-ray pulses are generated every $179 \mathrm{~ns}$ in this process. These characteristics of the beam result in scattering spectra which are cleaner and much simpler to interpret compared to Compton scattering experiments conducted with bremsstrahlung beams.

After passing through a collimator, the beam intensity is continuously monitored using a system of five thin plastic scintillator paddles [6] located upstream of the target. Recoil electrons and positrons produced in a thin metal radiator are detected in the paddles at a rate proportional to the incident flux. The absolute gamma ray flux measurement system is calibrated using a large $\mathrm{NaI}(\mathrm{Tl})$ detector which can measure the total incident flux of gamma rays with $100 \%$ efficiency. In order to maintain a relatively high live-time during the flux calibration, the incident flux on the NaI detector is reduced using high precision copper attenuators. The conversion factor between the observed paddle rate and the total number of detected gamma-rays is obtained by integrating the total counts in the NaI detector energy spectrum, knowing the total beam attenuation, and the paddle counting rate. This system provides a non-destructive continuous method of measuring the incident flux 
during production running. The Compton experiments use a circularly or linearly polarized beam with an intensity of $1-3 \times 10^{7} \mathrm{r} / \mathrm{s}$ on target.

A typical setup for Compton scattering experiments (without the recently added DIANA and BUNI detectors) is shown in figure 2. The beam is incident from the right of the figure indicated by the red arrow. The target cell, which has been described in detail in [8], is a $0.13 \mathrm{~mm}$ thick Kapton cylinder $20 \mathrm{~cm}$ long with a $4 \mathrm{~cm}$ diameter. The cell is enclosed in an aluminum vacuum can of diameter $46 \mathrm{~cm}$. The temperature in the target cell is monitored continuously throughout the experiment and is stable to within $0.2 \%$. The target density is known to better than $1 \%$. Scattering data are also collected with no liquid in the cell to assess the contribution from the windows and cell walls, which is typically found to be less than $15 \%$.

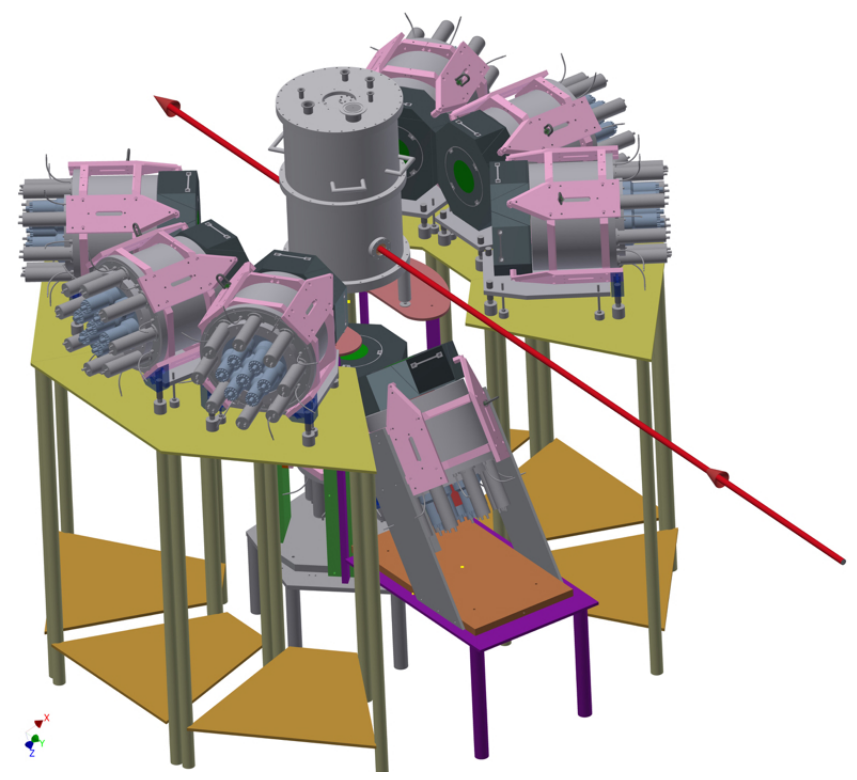

Figure 2: A Schematic of the Compton scattering experiment setup. This setup is used for both circularly and linearly polarized beams. For the case of experiments using linear polarization experiments, two or three detectors are positioned below the target to provide out-of-plane of polarization measurements.

Energy spectra are obtained using eight $\mathrm{NaI}(\mathrm{Tl})$ detectors positioned at angles ranging from $40^{\circ}$ to $159^{\circ}$ approximately $58 \mathrm{~cm}$ from the target center, as illustrated in Fig. 2. The core crystals, measuring $25.4 \mathrm{~cm}$ in diameter and ranging in length from $25.4 \mathrm{~cm}$ to $30.5 \mathrm{~cm}$, are surrounded by a segmented anti-coincidence shield consisting of eight $\mathrm{NaI}(\mathrm{Tl})$ crystals $7.5 \mathrm{~cm}$ thick and 30.5 $\mathrm{cm}$ in length. The acceptance cone of each core detector was defined by a $15 \mathrm{~cm}$ thick lead collimator. Each detector aperture is shielded using borated wax to suppress background neutrons. A high-precision survey of the experimental geometry is performed, and the measurements are incorporated into a GEANT simulation, which is used to determine the effective solid angle of each detector.

Detailed Monte Carlo simulations of the backgrounds, and Compton scattering events have been performed and validated with the measurements described in the previous section. For the case of deuteron, we have obtained $2.0 \%$ statistical accuracy in each detector with 300 hours of beam time and a flux of $3 \times 10^{7} \mathrm{\gamma} / \mathrm{s}$ on target. The systematic uncertainties contribute approximately 
$3 \%$ total error, dominated by the uncertainty in the flux, followed by the uncertainties in the target thickness, and the solid angle.

\section{Compton scattering on LHe, LD and LH targets}

Progress in calculating Compton scattering cross sections for light nuclei (namely, ${ }^{1} \mathrm{H},{ }^{2} \mathrm{H}$, ${ }^{3} \mathrm{He}$, and forthcoming ${ }^{4} \mathrm{He}$ ), which are sensitive to nucleon electromagnetic polarizabilities, has motivated numerous experiments at HIGS. A series of Compton scattering experiments on ${ }^{4} \mathrm{He},{ }^{2} \mathrm{H}$, and ${ }^{1} \mathrm{H}$ have been carried out and a Compton scattering cross section measurement on a cryogenic ${ }^{3} \mathrm{He}$ target is scheduled to start in the spring of 2020. A brief report on these experiments and preliminary results are presented here.

Due to relatively larger cross section, the Compton scattering program at HIGS was initiated using a cryogenic ${ }^{4} \mathrm{He}$ target [8]. Data were collected at gamma ray energies of 61 and $81 \mathrm{MeV}$. The 61-MeV measurement has been published [7] and the preliminary results from the $81-\mathrm{MeV}$ measurement are shown in Figure 3 and have been accepted for publication. The forward-backward angles asymmetry, or the backward angle peaking in this measurement is a clear signature of the polarizabiliy sensitivity of the cross section: the forward angles are sensitive to $\alpha+\beta$ (or the Baldin sum rule), and the backward angles are sensitive to $\alpha-\beta$. Theoretical efforts are underway to calculate this cross section and extract the isospin averaged nucleon polarizabilities.

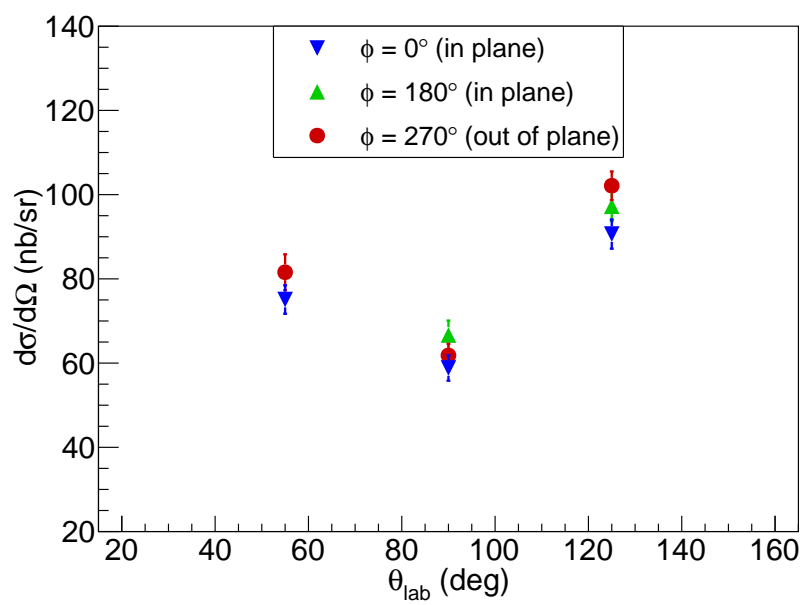

Figure 3: Plot of the Compton scattering cross section measured at HIGS at $81 \mathrm{MeV}$. Eight HINDA detectors were placed at three different scattering angles and three different azimuthal angles. This measurement was used to determine the instrumental azimuthal asymmetry correction for the linearly polarized Compton scattering measurement on the proton target which used the identical setup and immediately followed this experiment.

Compton scattering on deuterium has been carried out in a high-flux and low-resolution mode to provide better statistical accuracy. However, the combination of the beam energy spread and the detector energy resolution in this mode of operation does not allow distinction of the inelastic and elastic scattering contributions to the total cross section, as shown in figure 4. Resolving the two contributions (the inelastic peak is separated by the elastic peak by $2.25 \mathrm{MeV}$ ) requires improved 
beam energy resolution and detector with better energy resolution. To address these issues, we have acquired the two large, high energy-resolution detectors which were used in Compton experiments at LUND. These detectors, DIANA and BUNI, are described in detail in Ref. [3] and references therein. The HIGS data points are compared with previous measurements at LUND [3] and elsewhere. The theory curves are from $[1,2]$ for elastic deuteron Compton scattering. The dashed curves indicated central values displaced by \pm 2 standard units of $\delta(\alpha-\beta)$. At forward angles, the total scattering (elastic + inelastic) cross section nearly equals the predicted elastic-only cross section, whereas the backward angle data are higher than the theoretical elastic cross section. In a simple argument involving kinematics only, one can imagine that the backward angle has more inelastic contribution to the cross section due to a higher probability of large momentum transfer leading to quai- or inelastic scattering. Therefore, our data which includes both elastic and inelastic contributions are expected to agree with the theoretical predictions once our data are corrected (or new high resolution data are obtained). Also, there are current theoretical efforts underway which would calculate the polarizabilities from total scattering cross section for the deuteron. A set of high energy-resolution measurements are planned at HIGS using the DIANA and BUNI detectors. All of these efforts combined are expected to double the amount of data currently used in $\chi \mathrm{PT}$ extraction of neutron polarizabilities.

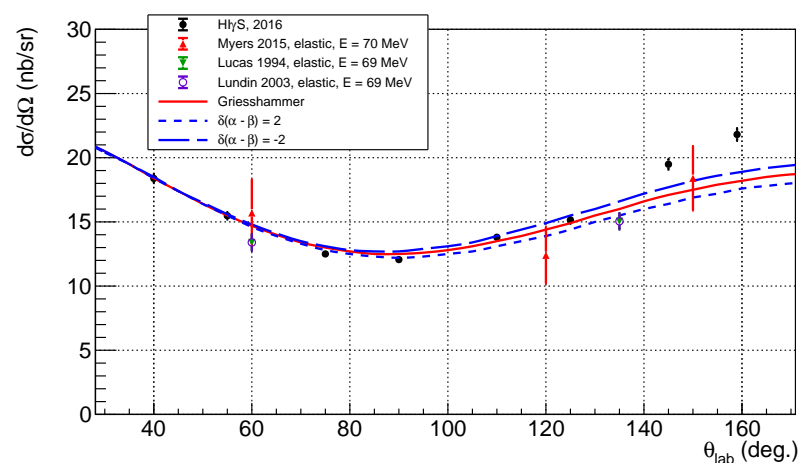

Figure 4: The deuteron Compton scattering total (elastic + inelastic) cross section at $65 \mathrm{MeV}$. The solid curve is the EFT prediction for the elastic scattering only. The dashed curves are obtained by displacing the central values of the polarizabilities by \pm 2 .

Data were also collected for proton Compton scattering at HIGS at gamma ray energy of 81 $\mathrm{MeV}$. Data were collected using both circular and linearly polarized gamma rays. Preliminary cross-section data from the circularly polarized Compton scattering is shown in figure 5. The linearly polarized data being analyzed.

\section{Upcoming Experiments}

Both DIANA and BUNI detectors have been commissioned at HIGS and will start acquiring high energy-resolution data on $\mathrm{L}^{3} \mathrm{He}$ and LD targets in the spring of 2020. The readiness to produce better energy resolution gamma-ray beams was recently tested at HIGS at gamma ray beam energy of $65 \mathrm{MeV}$. The collimator diameter was varied to study the effects on the beam energy resolution. A figure of merit was maximized between the interplay of the energy resolution and the flux. Based 


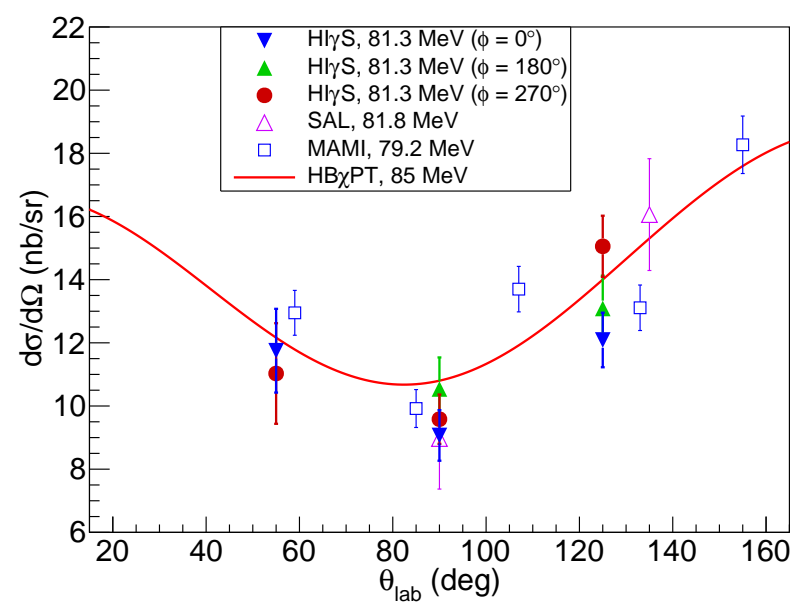

Figure 5: The proton Compton scattering cross section measured at HIGS at a gamma ray energy of $81 \mathrm{MeV}$. The filled triangles and circles represent data at various scattering angles as well as $\phi$ angle (only sensitive during linear polarization measurements). The open squares represent previous data near the energy of our measurement.

upon these studies, a $9 \mathrm{~mm}$ collimator has been selected for the Compton scattering measurements using DIANA/BUNI and high energy-resolution. An initial measurement using this setup and the DIANA detector for Compton scattering from ${ }^{12} \mathrm{C}$ target has demonstrated the capability of the system to resolve the contribution from inelastic scattering, from the $4.4 \mathrm{MeV}$ excited state, from the elastic scattering contribution. The cryogenic target is in the last stages of modifications to add a $1.4 \mathrm{~K}$ second-stage cooling and a new heat exchanger for liquifying ${ }^{3} \mathrm{He}$.

\section{References}

[1] Griesshammer, H., McGovern, J., \& Phillips, D. (2015). Compton scattering and nucleon polarisabilities in chiral EFT: The next steps. International Workshop on Chiral Dynamics.

[2] Griesshammer, H., McGovern, J., Phillips, D., \& Feldman, G. (2012). Using Effective Field Theory to analyse low-energy Compton scattering data from protons and light nuclei. Progress in Particle and Nuclear Physics, 67, 841-897.

[3] L.S. Myers, et al. (2014). Measurement of Compton Scattering from the Deuteron and an Improved Extraction of the Neutron Electromagnetic Polarizabilities. Phys. Rev. Lett., 113, 262506.

[4] Lensky, V.,\& Pascalutsa, V. (2010). Predictive powers of chiral perturbation theory in Compton scattering off protons. The European Physical Journal, C 65, 195-209.

[5] Weller, H. R., Ahmed, M. W., Gao, H., Tornow, W., Wu, Y., Gai, M., \& Miskimen, R. (2009). Research opportunities at the upgraded HIGS facility. Progress in Particle and Nuclear Physics, 62, 257-303.

[6] Pywell, R., Mavrichi, O., Wurtz, W., \& Wilson, R. (2009). Photon flux monitor for a mono-energetic gamma ray source. Nucl. Inst. Meth. Phys. Res., A 606, 517.

[7] Mark Sikora, et. al., (2017) Compton scattering from 4He at $61 \mathrm{MeV}$, Phys. Rev. C 96, 055209 
[8] Kendellen, D. P., Ahmed, M. W., Baird, E., Perreau, N., Wallace, P. W., Feldman, G., \& Weller, H. R. (2016). A Cryogenic Target for Compton Scattering Experiments at HIGS. Nucl. Inst. and Meth. in Phys. Res., A. 